\title{
Low-fat diet management strategy for chylothorax after pulmonary resection and lymph node dissection for primary lung cancer
}

Teruhisa Takuwa, MD, Junji Yoshida, MD, PhD, Shotaro Ono, MD, Tomoyuki Hishida, MD, PhD, Mitsuyo Nishimura, MD, PhD, Keiju Aokage, MD, PhD, and Kanji Nagai, MD, PhD

Objective: We reviewed our experience of iatrogenic chylothorax after pulmonary resection for primary lung cancer to evaluate a low-fat diet management strategy.

Methods: From October 2003 to March 2010, 1580 patients underwent lobectomy or greater resection and systematic mediastinal lymph node dissection for primary lung cancer at our institution. Chylothorax was diagnosed on the basis of chylous leakage from the chest tube and was confirmed by presence of triglycerides $(>110 \mathrm{mg} / \mathrm{dL})$ in the drainage fluid. We initially treated the patients with chylothorax conservatively with a low-fat diet (fat intake $<10 \mathrm{~g}$ /day). If chest tube drainage produced $>500 \mathrm{~mL}$ of chylous fluid during the first 24 hours after the initiation of the low-fat diet, surgical intervention was performed. If chest tube drainage produced $>300 \mathrm{~mL} /$ day of chylous fluid after 3 days of a low-fat diet, we performed pleurodesis by injecting a preparation of OK-432, a penicillin-treated lyophilized preparation of a Streptococcus strain into the thoracic cavity through a chest tube.

Results: Postoperative chylothorax developed in 37 patients (2.3\%), 33 men and 4 women, with a median age of 69 years (range, 44-84). The initial procedures were pneumonectomy in 1 patient and lobectomy in 36 patients. In 23 patients $(62 \%)$, their condition resolved with the low-fat diet only. A total of 10 patients underwent OK-432 pleurodesis, and 8 of these were cured with continuation of the low-fat diet. These 31 patients who responded to conservative treatment (84\%) resumed a normal diet at a median of 10 days (range, 5-27) after the chylothorax diagnosis. The remaining 6 patients $(16 \%)$ underwent reoperation and were discharged at a median of 18 days (range, 14-33) after the initial surgery.

Conclusions: A low-fat diet and OK-432 pleurodesis achieved positive results in $>80 \%$ of patients with chylothorax after pulmonary resection with systematic mediastinal lymph node dissection within 4 weeks after the initial surgery. More than $500 \mathrm{~mL}$ of chylous fluid during the first 24 hours after the initiation of the low-fat diet was valid as an indication of the need for surgical intervention. (J Thorac Cardiovasc Surg 2013;146:571-4)

Earn CME credits at

http://cme.ctsnetjournals.org

Postoperative chylothorax is a relatively rare, but wellknown, complication of thoracic surgery. However, because of its rare occurrence, its management strategy has not been well established, and a practical strategy is needed.

In 2002, we reviewed our experiences with iatrogenic chylothorax after pulmonary resection for lung cancer to evaluate our treatment strategy and to identify the factors that predicted the need for reoperation. Although most

\footnotetext{
From the Division of Thoracic Surgery, Department of Thoracic Oncology, National Cancer Center Hospital East, Kashiwa, Chiba, Japan.

Disclosures: Authors have nothing to disclose with regard to commercial support.

Received for publication Oct 1, 2012; revisions received Feb 14, 2013; accepted for publication April 18, 2013; available ahead of print June 13, 2013.

Address for reprints: Junji Yoshida, MD, PhD, Division of Thoracic Surgery, Department of Thoracic Oncology, National Cancer Hospital East, 6-5-1, Kashiwanoha, Kashiwa, Chiba 277-8577, Japan (E-mail: jyoshida@east.ncc.go.jp). $0022-5223 / \$ 36.00$

Copyright (c) 2013 by The American Association for Thoracic Surgery http://dx.doi.org/10.1016/j.jtcvs.2013.04.015
}

patients recovered fully with a conservative strategy of complete oral intake cessation and total parenteral nutrition (TPN), early surgical intervention was indicated if chest tube drainage of $>500 \mathrm{~mL}$ of chylous fluid was observed during the first 24 hours after complete oral intake cessation and the initiation of TPN. ${ }^{1}$ Because of recent reports that have advocated a low-fat diet for reducing chylous flow, ${ }^{2,3}$ we developed a low-fat diet management strategy for postoperative patients with chylothorax and collected the data regarding the outcomes of patients with iatrogenic chylothorax after pulmonary resection with systematic mediastinal lymph node dissection for lung cancer.

\section{METHODS}

From October 2003 through March 2010, 1580 patients underwent lobectomy or greater resection plus systematic lymph node dissection for primary lung cancer at our hospital. A diagnosis of chylothorax was determined by chylous leakage from a chest tube and was confirmed by the presence of an elevated triglyceride level $(>110 \mathrm{mg} / \mathrm{dL})$ in the drainage fluid. The drainage volume was not considered in the chylothorax diagnosis. Postoperative chylothorax developed in 37 patients $(2.3 \%)$, and these patients constituted the subjects of the present study.

We treated these patients conservatively with a low-fat diet (fat $<10$ $\mathrm{g} /$ day), prohibiting any consumption of fat-containing food or drink, and 


\section{Abbreviation and Acronym \\ $\mathrm{TPN}=$ total parenteral nutrition}

measured the amount of chylous drainage fluid (Figure 1). Diet compliance was monitored by our hospital nutritionist. If the volume of chylous fluid observed was $>500 \mathrm{~mL}$ during the first 24 hours after the initiation of conservative therapy, immediate reoperation was performed. If the volume remained $>300 \mathrm{~mL} /$ day 3 days after the chylothorax diagnosis, we performed pleurodesis by injecting a mixture of 10 Klinische Einheit units of OK-432 (a heat- and penicillin-treated lyophilized preparation of the Su strain of Streptococcus pyogenes A3; Picibanil; Chugai Pharmaceutical, Tokyo, Japan), $10 \mathrm{~mL}$ of $1 \%$ lidocaine, and $50 \mathrm{~mL}$ isotonic saline into the thoracic cavity through a chest tube. If the chest tube drainage was $<300 \mathrm{ml} /$ day after 3 days, the patients continued the low-fat diet. If the chylothorax had not been cured within about 2 weeks with this conservative strategy, surgical intervention was performed. A patient was considered cured when the chest tube drainage fluid was serous, the volume was $<200 \mathrm{~mL} / \mathrm{day}$, and if the fluid did not become chylous even after the patient had eaten fatty ice cream. We challenged with ice cream on the first day the chest tube output was $<200 \mathrm{ml} /$ day and serous.

The present study was a retrospective analysis of prospectively collected patient data after the diagnosis of chylothorax from October 2003 to March 2010. Data from the patients with chylothorax were collected prospectively. The diagnosis and management protocol was implemented in 2003 and used uniformly for all eligible patients, whose operations were performed by the 4 attending surgeons, for the entire study period. The medical records of these patients were reviewed for age, gender, disease laterality, surgical procedures, chest tube drainage amount, treatment course, and length of hospital stay. The institutional review board approved the data collection and analyses and waived the need to obtain written informed consent from each patient in March 2011.

\section{RESULTS}

Of the 37 patients, 33 were men and 4 were women. Their age ranged from 44 to 88 years (median, 69). The initial procedure was right pneumonectomy in 1 patient $(3 \%)$ and lobectomy in 36 patients $(97 \%)$. Lobectomy on the right side was performed in 27 patients $(73 \%)$, including 20 upper lobectomies (54\%), 5 lower lobectomies (14\%), and 2 middle lobectomies $(5 \%)$. Lobectomy on the left side was performed in 9 patients $(24 \%)$, including 6 upper lobectomies $(16 \%)$ and 3 lower lobectomies $(8 \%$; Table 1$)$. Three patients received induction therapy ( 1 chemotherapy and 2 concurrent chemoradiotherapy). Initial surgery was performed with video thoracoscopic assistance in $34 \mathrm{pa}-$ tients ( $84 \%$; Table 1$)$. The pathologic lymph node metastases were stage $\mathrm{pN} 0$ in 31 patients $(84 \%)$, pN1 in 3 patients $(8 \%)$, and $\mathrm{pN} 2$ in 3 patients $(8 \%)$.

Of these 37 patients, $4(11 \%)$ underwent immediate surgery, because the volume of chest tube drainage chylous fluid was $>500 \mathrm{~mL}$ during the first 24 hours of a low-fat diet (Figure 2).

The remaining 33 patients continued with the low-fat diet, and in $23(62 \%)$, the condition resolved. However, because of a continuous chylous fluid volume of $>300 \mathrm{~mL} /$ day 3

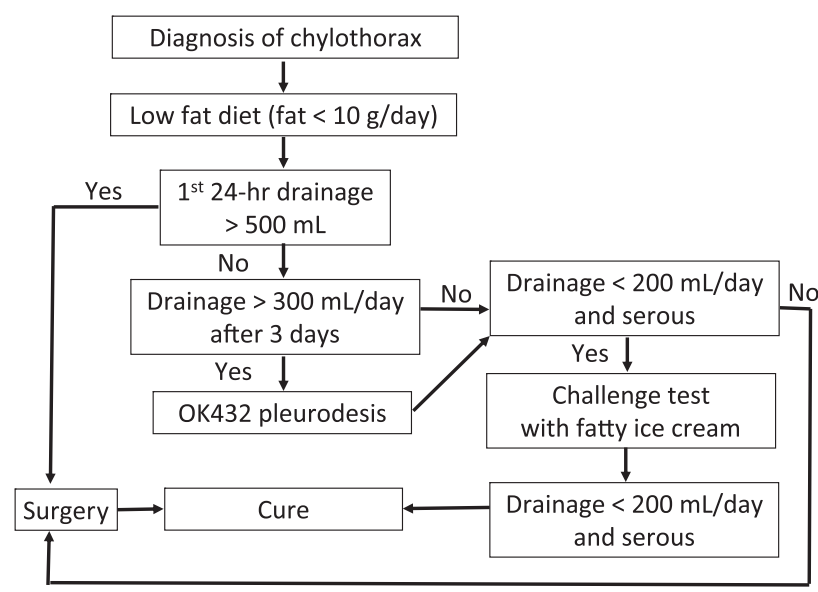

FIGURE 1. Low-fat diet management strategy for postoperative chylothorax. OK-432 refers to a heat- and penicillin-treated lyophilized preparation of the Su strain of Streptococcus pyogenes A3.

days after the chylothorax diagnosis, 10 patients $(27 \%)$ underwent pleurodesis with the OK-432 mixture. After pleurodesis, the condition resolved in 8 patients $(22 \%)$. The remaining 2 patients underwent reoperation, because their chylothorax had not been cured by approximately 2 weeks after the initiation of the current conservative therapy.

In all, 6 patients $(16 \%)$ underwent reoperation (4 immediately and 2 after pleurodesis) with thoracoscopic assistance. The initial surgery of these 6 patients was right-sided lobectomy with mediastinal lymph node dissection. Injury to the thoracic duct was indentified in 3 patients, and the injuries were sutured and covered with a TachoComb fibrinogen tissue sealing sheet (CSL Behring, Tokyo, Japan). In all cases, the thoracic duct was ligated at the level of the diaphragm. After reoperation, the chest tube drainage decreased, and the chylothorax was cured in 5 patients at a median of 10 days (range, 3-10) after reoperation and a median of 13 days (range, 6-16) after the initial surgery. However, 1 patient required additional pleurodesis because of continued chylous leakage. His chylothorax was diagnosed as cured and the chest tube was removed 21 days after the initial surgery (18 days after the chylothorax surgery).

In total, postoperative chylothorax was conservatively cured in 31 patients $(84 \%)$ and their chest tube was removed a median of 10 days (range, 5-20) after the initial surgery. In these 31 patients, 8 patients underwent pleurodesis and were cured without surgery. Their chest tubes were removed a median of 13 days (range, 5-18) after their initial surgery. In the remaining 23 patients treated with the lowfat diet alone, their chest tubes were removed at a median of 8 days (range, 5-20). The remaining 6 patients (16\%) underwent reoperation, the condition resolved, and their chest tube was removed a median of 13 days (range, 6-21) after the initial surgery. No 30-day or in-hospital deaths occurred in any patients. Finally, 2 patients developed arrhythmia, 2 
TABLE 1. Patient characteristics

\begin{tabular}{|c|c|}
\hline Characteristic & Value \\
\hline \multicolumn{2}{|l|}{ Age (yr) } \\
\hline Median & 69 \\
\hline Range & $44-88$ \\
\hline \multicolumn{2}{|l|}{ Gender (n) } \\
\hline Men & $33(89)$ \\
\hline Women & $4(11)$ \\
\hline \multicolumn{2}{|l|}{ Induction therapy (n) } \\
\hline Yes & $3(8)$ \\
\hline Concurrent chemoradiotherapy & 2 \\
\hline Chemotherapy & 1 \\
\hline No & $34(84)$ \\
\hline \multicolumn{2}{|l|}{ Initial operation (n) } \\
\hline Lobectomy on right & $27(73)$ \\
\hline Upper lobectomy & $20(54)$ \\
\hline Lower lobectomy & $5(14)$ \\
\hline Middle lobectomy & $2(5)$ \\
\hline Lobectomy on left & $9(24)$ \\
\hline Upper lobectomy & $6(16)$ \\
\hline Lower lobectomy & $3(8)$ \\
\hline Right pneumonectomy & $1(3)$ \\
\hline \multicolumn{2}{|l|}{ Video thoracoscopic assistance (n) } \\
\hline Yes & $34(84)$ \\
\hline No & $3(8)$ \\
\hline \multicolumn{2}{|l|}{ Pathologic N stage (n) } \\
\hline No & $31(84)$ \\
\hline N1 & $3(8)$ \\
\hline $\mathrm{N} 2$ & $3(8)$ \\
\hline \multicolumn{2}{|l|}{ Pathologic stage (UICC 6th edition) (n) } \\
\hline IA & $14(38)$ \\
\hline IB & $9(24)$ \\
\hline IIA & $1(3)$ \\
\hline IIB & $7(19)$ \\
\hline IIIA & $4(11)$ \\
\hline IIIB & $2(5)$ \\
\hline
\end{tabular}

Data in parentheses are percentages. UICC, International Union Against Cancer.

acute empyema, 1 delirium, and 1 atelectases. All these patients were treated conservatively and were cured.

\section{DISCUSSION}

Chyle duct injury with resulting postoperative chylothorax is a relatively rare complication of thoracic surgery. Iatrogenic chylothorax occurs in $0.25 \%$ to $0.5 \%$ of all thoracic surgery cases and has also been described in association with neck dissection, cardiac surgery, pulmonary resection, and esophagectomy. ${ }^{2-4}$ In the present study, the incidence rate of iatrogenic chylothorax was much higher than that described in previous reports. This might have been because all the patients in the present series had undergone systematic mediastinal lymph node dissection, as previously described..$^{5}$

The nutritional state of postoperative patients with chylothorax can deteriorate quickly, resulting in poor immunologic status and thoracic infection; therefore, the

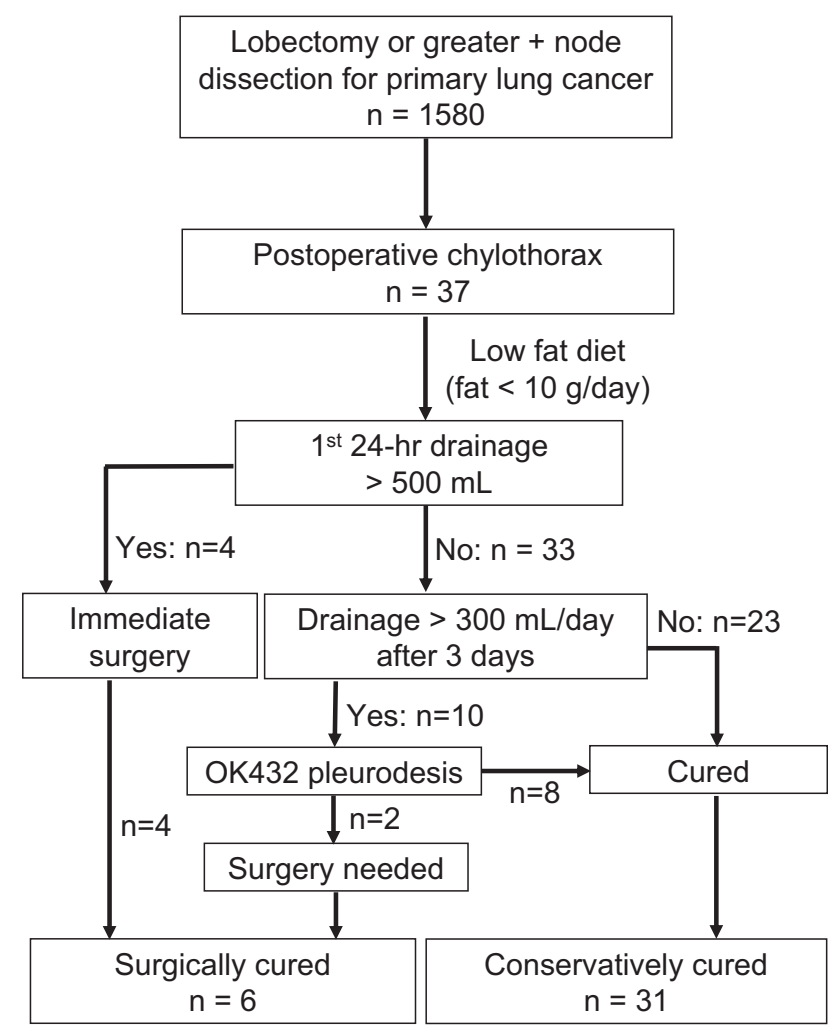

FIGURE 2. Chylothorax management strategy and outcomes.

condition needs to be rapidly and effectively managed. The strategy of conservative treatment includes the reduction of the chyle flow, inflating the lung, decreasing the dead space in the thoracic cavity, nutritional support, and the prevention of septic complications. ${ }^{6}$ Thoracic duct chyle flow increases after eating, in particular after a high-fat meal. ${ }^{7}$ The volume of chyle flow can be reduced by avoiding fat-containing enteral nutrition. ${ }^{8}$ Therefore, complete oral intake cessation, with TPN or a low-fat diet, has often been recommended. However, TPN can be associated with complications such as venous line infection and mucosal integrity disorder. In contrast, oral intake and enteral nutrition have been reported to be safe and more natural, with fewer complications. ${ }^{9,10}$ Despite these reported advantages of enteral feeding, many studies of the conservative management of chylothorax have suggested the use of TPN was more effective than a low-fat diet. $^{3,11,12}$ We also reported successful chylothorax management using TPN, with complete oral intake cessation in $2002 .^{1}$

However, several researchers have recently reported the advantages of a low-fat diet in reducing the thoracic duct flow of chyle. ${ }^{13-15}$ On the basis of their findings and our previously reported experience, ${ }^{1}$ we have developed a low-fat diet management strategy for postoperative chylothorax, with surgical intervention if the chest tube discharge 
during the first 24 hours is $>500 \mathrm{~mL}$. This conservative strategy of a low-fat diet cured 31 patients $(84 \%)$ at a median of 10 days (range, 5-20) after the initial lung resection. Compared with our previous strategy of complete oral intake cessation and TPN, with which $81 \%$ of the patients were cured at a median of 8 days (range, 4-35) after the chylothorax diagnosis, ${ }^{1}$ we believe this new strategy is also effective, with the least amount of burden to the patient. Of the 10 patients who required pleurodesis with the OK-432 mixture, the condition was cured in $8(80 \%)$ without surgical intervention. Together with our previous report, in which 13 of the 15 patients $(87 \%)$ receiving OK-432 pleurodesis were cured, we have demonstrated that pleurodesis is an effective adjunct in patients in whom excessive drainage discharge persists even after 3 days of reduced thoracic duct flow.

Surgical repair should be considered only when it has become clear that conservative treatment has failed. The clinical parameters that prompt surgical intervention have varied among reports. ${ }^{16,17}$ We previously reported that chest tube drainage discharge of $>500 \mathrm{~mL}$ during the first 24 hours after complete oral intake cessation and TPN predicted continued leakage, and, therefore, an immediate reoperation might be indicated. ${ }^{1}$ In the present study, we considered surgical intervention necessary if the chest tube drainage during the first 24 hours after the initiation of a low-fat diet was $>500 \mathrm{~mL}$, or if the patient's chylothorax was not cured within about 2 weeks with conservative treatment. We performed reoperation in 6 patients $(16 \% ; 4$ immediately and 2 after pleurodesis). Even in these surgically treated patients, cure was achieved and the chest tube was removed at a reasonable median of 13 days (range, 6-21) from the initial surgery.

The present study had some limitations. In particular, the patients were drawn from only 1 institution and from a generally homogenous ethnic group. Furthermore, although we achieved $80 \%$ cure, the number of patients was small. Because no control group was included, it would be difficult to draw strong conclusions about the efficacy of our approach.

However, in conclusion, $>80 \%$ of chylothorax cases after pulmonary resection with mediastinal lymph node dissection could be cured with our low-fat diet management strategy. Reoperation was indicated for $<20 \%$ of the patients, which minimized the overall number of days needed until a cure was obtained. These results were similar to the outcomes of our previous strategy of TPN and complete oral intake cessation. ${ }^{1}$ Our chylothorax management strategy with a low-fat diet was effective, with a minimal patient burden.

We are indebted to Assistant Professor Rod Turner of Toho University Medical School and Professor J. Patrick Barron, Chair of the Department of International Medical Communications at Tokyo Medical University, for their editorial review of our report.

\section{References}

1. Shimizu K, Yoshida J, Nishimura M, Takamochi K, Nakahara R, Nagai K. Treatment strategy for chylothorax after pulmonary resection and lymph node dissection for lung cancer. J Thorac Cardiovasc Surg. 2002;124:499-502.

2. McGrath EE, Blades Z, Anderson PB. Chylothorax: aetiology, diagnosis and therapeutic options. Respir Med. 2010;104:1-8.

3. Nair SK, Petko M, Hayward MP. Aetiology and management of chylothorax in adults. Eur J Cardiothorac Surg. 2007;32:362-9.

4. Cerfolio RJ, Allen MS, Deschamps C, Trastek VF, Pairolero PC. Postoperative chylothorax. J Thorac Cardiovasc Surg. 1996;112:1361-5; discussion 5-6.

5. Naruke T. Mediastinal lymph node dissection. In: Shiels TW, ed. General thoracic surgery. Philadelphia: Lea \& Febiger; 1995:469-80.

6. Merrigan BA, Winter DC, O'Sullivan GC. Chylothorax. Br J Surg. 1997;84: 15-20.

7. Paes ML, Powell H. Chylothorax: an update. Br J Hosp Med. 1994;51:482-90.

8. Blalock A, Cunningham RS, Robinson CS. Experimental production of chylothorax by occlusion of the superior vena cava. Ann Surg. 1936;104:359-64.

9. Preiser JC, Berre J, Carpentier Y, Jolliett P, Pichard C, Van Gossum A, et al. Management of nutrition in European intensive care units: results of a questionnaire. Working Group on Metabolism and Nutrition of the European Society of Intensive Care Medicine. Intensive Care Med. 1999;25:95-101.

10. Yi F, Ge L, Zhao J, Lei Y, Zhou F, Chen Z, et al. Meta-analysis: total parenteral nutrition versus total enteral nutrition in predicted severe acute pancreatitis. Intern Med. 2012;51:523-30.

11. Fernandez Alvarez JR, Kalache KD, Grauel EL. Management of spontaneous congenital chylothorax: oral medium-chain triglycerides versus total parenteral nutrition. Am J Perinatol. 1999;16:415-20.

12. Orringer MB, Bluett M, Deeb GM. Aggressive treatment of chylothorax complicating transhiatal esophagectomy without thoracotomy. Surgery. 1988;104: $720-6$.

13. Browse NL, Allen DR, Wilson NM. Management of chylothorax. Br J Surg. 1997;84:1711-6.

14. Leibovitch I, Mor Y, Golomb J, Ramon J. The diagnosis and management of postoperative chylous ascites. J Urol. 2002;167(2 Pt 1):449-57.

15. Su IC, Chen CM. Spontaneous healing of retroperitoneal chylous leakage following anterior lumbar spinal surgery: a case report and literature review. Eur Spine J. 2007;16(suppl 3):332-7.

16. Dugue L, Sauvanet A, Farges O, Goharin A, Le Mee J, Belghiti J. Output of chyle as an indicator of treatment for chylothorax complicating oesophagectomy. Br J Surg. 1998;85:1147-9.

17. Marts BC, Naunheim KS, Fiore AC, Pennington DG. Conservative versus surgical management of chylothorax. Am J Surg. 1992;164:532-4; discussion 4-5. 